\title{
REVISED LINE PROFILE FUNCTION FOR HYDROGENIC SPECIES
}

\author{
A. Sapar and R. Poolamäe \\ Tartu Observatory,61602 Tõravere,Estonia; sapar@aai.ee, praivo@aai.ee
}

Received: 2012 June 1; accepted: 2012 June 18

\begin{abstract}
Analytical series expansions for the hydrogenic spectral line profile functions are derived starting from the three single expressions, obtained by integrating twice the convolution of the Holtsmark, Lorentz and Doppler line profile functions. We get well converging series expansions for the line wings and centers by reducing the number of arguments in the profile function by one, introducing the module of the Holtsmark and Lorentz profiles as a new argument. In the intermediate part of the line, the parabolic cylinder functions expressed via the confluent hypergeometric series, are used. The multi-component Stark splitting of the hydrogenic spectral lines and the modeled stochastic electron transitions in the electric field of the adjacent ions generate wide Doppler plateaux at the line centers, with the characteristic widths estimated from the fit to the characteristic width of the Holtsmark profile. This additional Doppler broadening of the line profile function removes the central dip typical to the Holtsmark profile.
\end{abstract}

Key words: atomic processes - line: profiles - opacity - radiative transfer stars: atmospheres

\section{INTRODUCTION}

In the present paper we propose a new and more compact version of the analytical formulae for line profile functions of the hydrogen atoms and hydrogenic ions. However, different series expansion formulae are needed for the line wings, at the line center and in its intermediate region.

The line profile functions of hydrogenic particles, in addition to thermal (Doppler) and damping (Lorentz) contributions (their convolution is named the Voigt function), undergo the linear Stark effect. Its contribution due to electrostatic fields of adjacent ions we describe in the approximation of the Holtsmark profile. We treat hydrogenic line profile functions as a convolution of the mentioned three contributing functions, corresponding to different physical processes.

A very complicated problem is to take correctly into account the multi-component linear Stark effect splitting the hydrogenic spectral lines and the quantum electrodynamic contribution of the adjacent ions, described by the model microfield method (MMM), which demands computation of individual line profile tables. Here we try to take the effect into account fitting it to additional Doppler broadening what is adequate both in the line centers and wings. 


\section{STARTING FORMULAE}

We start from the Holtsmark profile type integrals with the additional two parameters, describing the Lorentz and Doppler contributions. Such expressions were derived by Sapar \& Kuusik (1973) and further studied by Sapar et al. (2006, 2009). In the paper by Sapar and Kuusik, the double integration of triple integrals was carried out and the convolution of the Holtsmark and Voigt profiles has been reduced to a sum of three integrals. These integrals can be treated as describing dominantly the Holtsmark, Lorentz and Doppler contributions, namely

$$
\Phi(\beta)=\chi(\beta)+\Lambda(\beta)+\Delta(\beta),
$$

where

$$
\begin{aligned}
& \chi(\beta)=\frac{2}{\pi} \int_{0}^{\infty} \beta x \sin (\beta x) \varepsilon(x) d x, \\
& \Lambda(\beta)=\frac{2 L}{\pi} \int_{0}^{\infty} x \cos (\beta x) \varepsilon(x) d x
\end{aligned}
$$

and

$$
\Delta(\beta)=\frac{4 D^{2}}{\pi} \int_{0}^{\infty} x^{2} \cos (\beta x) \varepsilon(x) d x .
$$

In these expressions the total exponential term

$$
\varepsilon(x)=\exp \left(-x^{3 / 2}-L x-D^{2} x^{2}\right),
$$

where the constants $D$ and $L$ are the ratios

$$
D=\frac{\Delta \nu_{D}}{2 \Delta \nu_{S}}, \quad L=\frac{\Delta \nu_{L}}{\Delta \nu_{S}}
$$

Here the quantities $\Delta \nu_{D}$ and $\Delta \nu_{L}$ are widths of the Doppler and Lorentz line profiles, respectively, $\Delta \nu_{S}$ is the characteristic width of the Stark profile and the argument of spectral line profile function $\beta=\Delta \nu / \Delta \nu_{S}$. The Doppler and the Lorentz profile widths are

$$
\Delta \nu_{D}=\frac{\nu_{0}}{c} \sqrt{\frac{2 k T}{m_{e}}}, \quad \Delta \nu_{L}=\gamma_{L} / 2
$$

Here $\gamma_{L}$ is the Lorentz damping constant in $\nu$ units. The characteristic Stark profile width $\Delta \nu_{S}$ has been found averaging over the Stark shift components by different methods. Presently we use the formula which follows from the equation of Griem (1960), given also in the book by Sobelman (1963, p. 504, Eq. 38.13). The proportionality coefficient between the Stark shift $\Delta \nu_{S}$ and the averaged electric field strength $E_{0}$ is

$$
B=\frac{3^{2 / 3} \hbar}{8 \pi Z e m}\left(n_{u}^{2}-n_{l}^{2}\right)
$$


where $n_{u}$ is the principal quantum number of the upper state and $n_{l}$ - of the lower state. Evidently, $E_{0}$ can be defined for hydrogenic particles of the nuclear charge $Z e$ by

$$
E_{0}=\frac{Z e}{R_{0}^{2}}, \quad N^{-1}=\frac{4 \pi R_{0}^{3}}{3} .
$$

Consequently

$$
\Delta \nu_{S}=B E_{0}=\frac{\hbar\left(n_{u}^{2}-n_{l}^{2}\right)}{2(4 \pi)^{1 / 3} m} N^{2 / 3} .
$$

Now all parameters in the starting equations are specified.

\section{AUXILIARY INTEGRALS}

Using a generating integral

$$
K(\beta)=\int_{0}^{\infty} \cos (\beta x) \varepsilon(x) d x,
$$

we can write

$$
\chi(\beta)=-\frac{2 \beta}{\pi} \frac{d K(\beta)}{d \beta}
$$

and

$$
\Delta(\beta)=-\frac{4 D^{2}}{\pi} \frac{d^{2} K(\beta)}{d \beta^{2}} .
$$

For more compact treatment of the series expansions of the exponential function $\varepsilon(x)$, it is useful to introduce a complex phase factor for $\beta$ in the needed integrals

$$
K_{j}(\beta)=\int_{0}^{\infty} x^{j} \exp (i \beta x) \varepsilon(x) d x=c_{j}(\beta)+i s_{j}(\beta),
$$

where the complex phase factor is

$$
\exp (i \beta x)=\cos (\beta x)+i \sin (\beta x) .
$$

Thus we get

$$
\begin{aligned}
& \chi(\beta)=\frac{2}{\pi} \beta s_{1}(\beta), \\
& \Lambda(\beta)=\frac{2 L}{\pi} c_{1}(\beta)
\end{aligned}
$$

and

$$
\Delta(\beta)=\frac{4 D^{2}}{\pi} c_{2}(\beta) .
$$

The argument of spectral line profile $\beta$ in the phase factor can be joined with $L$ in exponent as a complex exponent and thereafter integrations can be carried out with its use. If we ignore the exponential function in the Holtsmark line 
profile formula, then the needed analytical integrals can be represented in the form (Gradshtein \& Ryzhik 1962, p. 494, formula 3.897):

$$
I(z)=\int_{0}^{\infty} \exp \left(-D^{2} x^{2}-\gamma x\right) d x=\frac{\sqrt{\pi}}{2 D}\left\{\exp \left(z^{2}\right) \operatorname{erfc}(z)\right\},
$$

where the complex spectral line arguments

$$
\gamma=L-i \beta, \quad z=\frac{\gamma}{2 D}
$$

the complex probability integral

$$
\operatorname{erf}(z)=\frac{2}{\sqrt{\pi}} \int_{0}^{z} \exp \left(-t^{2}\right) d t
$$

and

$$
\operatorname{erfc}(z)=1-\operatorname{erf}(z)
$$

The derivatives $d^{j} I / d L^{j}$ describe analytical solutions to the profile integrals, which have additional integer power factor $x^{j}$ in the integrand of (14). Such integrals with the integer value $3 j$ appear if we use the series expansion of the exponent function $\exp \left(-x^{3 / 2}\right)$ in the Holtsmark profile.

The complex probability integral can be expressed as the series expansion

$$
\operatorname{erf}(z)=\frac{2}{\sqrt{\pi}} \exp \left(-z^{2}\right) \sum_{k=0}^{\infty} \frac{2^{k} z^{2 k+1}}{(2 k+1) ! !} .
$$

This formula holds for all values of the argument $z$, but it is rapidly convergent only at its small values. For spectral line wings at large values of argument $\beta$, where this series expansion converges very slowly, it can be expressed by the following asymptotic form (Gradshtein \& Ryzhik 1962, formula 9.243.2)

$$
\operatorname{erfc}(z)=\frac{1}{\pi} \exp \left(-z^{2}\right) \sum_{k=0}^{\infty} \frac{(-1)^{k} \Gamma(k+1 / 2)}{z^{2 k+1}} .
$$

It is evident that at large $z$ values the exponential terms with $z^{2}$ in the integral $I(z)$, formula (19), cancel one another.

\section{CONVOLVED PROFILE IN LINE WINGS}

Now let us consider the convolved spectral line profile function by its series expansion at large argument values, i.e. in the far wings of spectral lines. Therefore, differently from our former study (Sapar et al. 2006), we conserve the Lorentz exponent in the spectral line wings, i.e. at large $\beta$ values, but use the series expansions for the Doppler and Holtsmark contributions in the exponent functions.

Thus, for the line wings we obtain the integrals of the form

$$
P_{\mu}(\gamma)=\int_{0}^{\infty} x^{\mu-1} \exp (-\gamma x) d x=\frac{\Gamma(\mu)}{\gamma^{\mu}}=\frac{\Gamma(\mu)}{\rho^{\mu}} \exp (i \mu \varphi),
$$


where we took into account that

$$
\gamma=L-\mathrm{i} \beta=\rho \exp (-i \varphi)
$$

where the module $\rho$ and the phase angle $\varphi$ of the complex number $\gamma$ are given by

$$
\rho=\left(L^{2}+\beta^{2}\right)^{1 / 2}, \quad \varphi=\arcsin \left(\frac{\beta}{\rho}\right)
$$

For the line wings it is useful to express the terms corresponding to the series expansion in the form

$$
P_{\mu}(\gamma)=C_{\mu}(\rho, \varphi)+i S_{\mu}(\rho, \varphi)
$$

where

$$
S_{\mu}(\rho, \varphi)=\int_{0}^{\infty} \exp (-L x) x^{\mu-1} \sin (\beta x) d x=\frac{\Gamma(\mu)}{\rho^{\mu}} \sin (\mu \varphi)
$$

and

$$
C_{\mu}(\rho, \varphi)=\int_{0}^{\infty} \exp (-L x) x^{\mu-1} \cos (\beta x) d x=\frac{\Gamma(\mu)}{\rho^{\mu}} \cos (\mu \varphi) .
$$

In these series expansions

$$
\mu=j+\frac{3 n}{2}+2 m
$$

where $j$ is the contribution outside of the series expansion, $n$ corresponds to the series expansion of the Holtsmark exponent and $m$ - to the series expansion of the Doppler exponent. This general form of integrals in the line wing profile expansion of the Holtsmark and Doppler exponents converges well only at large values of $\beta$. The line profile integrals can be now written in the form

$$
\begin{array}{ll}
\chi(\beta)=\frac{2}{\pi \beta} \sum_{n=0}^{\infty} \sum_{m=0}^{\infty} \frac{(-1)^{m+n}}{n ! m !} D^{2 m} S_{\mu}(\rho, \varphi), & j=1 \\
\Lambda(\beta)=\frac{2 L}{\pi \beta^{2}} \sum_{n=0}^{\infty} \sum_{m=0}^{\infty} \frac{(-1)^{m+n}}{n ! m !} D^{2 m} C_{\mu}(\rho, \varphi), & j=1
\end{array}
$$

and

$$
\Delta(\beta)=\frac{4 D^{2}}{\pi \beta^{3}} \sum_{n=0}^{\infty} \sum_{m=0}^{\infty} \frac{(-1)^{m+n}}{n ! m !} D^{2 m} C_{\mu+1}(\rho, \varphi), \quad j=2 .
$$

In the far wings of spectral line, i.e., if $\beta \gg L$, the phase angle $\varphi$ tends to $\pi / 2$. Consequently, in the far wings the series expansion depends polynomially on $1 / \rho$. The convergence relative to the Doppler width is rapid if $D<1$, i.e. in the deep layers of stellar atmospheres. 


\section{CONVOLVED PROFILE AT LINE CENTERS}

In order to study the hydrogenic line profile in the central region of the spectral line profile, it is suitable to conserve the exponential function of the Holtsmark profile and to introduce for it the notation

$$
\eta(x)=\exp \left(-x^{3 / 2}\right)
$$

Now it is useful to introduce the complex integrals

$$
B_{k}(\gamma)=\int_{0}^{\infty} x^{k} \exp (-\gamma x) \eta(x) d x
$$

Applying the Moivre formula for the series expansion of the complex exponential function, we obtain

$$
\exp (-\gamma x)=\sum_{n=0}^{\infty} \frac{(-\rho x)^{n}}{n !} \exp (-\mathrm{i} n \varphi)
$$

From here we obtain a dependence of the line profile function on $\rho$ and $\varphi$, which for the line center, $\beta<L$, is a polynomial expression relative to $L$.

Introducing variable $y=x^{3 / 2}$ and using the series expansion

$$
\exp \left(-D^{2} x^{2}\right)=\sum_{m=0}^{\infty} \frac{(-1)^{m} D^{2 m}}{m !} x^{2 m}
$$

we get the values of the Holtsmark exponent integrals in the form

$$
G_{k}=\int_{0}^{\infty} x^{k} \exp \left(-x^{3 / 2}\right) d x=\frac{2}{3} \int_{0}^{\infty} y^{(2 k-1) / 3} \exp (-y) d y=\frac{2}{3} \Gamma\left(\frac{2 k+2}{3}\right),
$$

where in $x^{k}$ the exponent $k$ is given by

$$
k=j+n+2 m .
$$

Using the series expansions of $\exp (-\gamma x)$ and $\exp \left(-D^{2} x^{2}\right)$, we obtain for the needed integrals $K_{j}$, equation (14), the series expansion

$$
\exp \left(-\gamma x-D^{2} x^{2}\right)=\sum_{m=0}^{\infty} \sum_{n=0}^{\infty}(-1)^{m+n} \frac{D^{2 m}}{m !} \frac{\rho^{n}}{n !} \exp (-\mathrm{i} n \varphi) .
$$

Now in a compact form we can write

$$
K_{j}=\sum_{n=0}^{\infty} I_{n+j}\left(C_{n}-\mathrm{i} S_{n}\right),
$$

where

$$
I_{n+j}=\sum_{m=0}^{\infty}(-1)^{n+m} G_{j+n+2 m} \frac{D^{2 m}}{m !},
$$




$$
C_{n}=\frac{\rho^{n} \cos (n \varphi)}{n !}
$$

and

$$
S_{n}=\frac{\rho^{n} \sin (n \varphi)}{n !}
$$

Thus we obtain

$$
\begin{aligned}
& \chi(\beta)=\frac{2}{\pi} \beta \sum_{n=0}^{\infty} I_{n+1} S_{n}, \\
& \Lambda(\beta)=\frac{2 L}{\pi} \sum_{n=0}^{\infty} I_{n+1} C_{n}
\end{aligned}
$$

and

$$
\Delta(\beta)=\frac{4 D^{2}}{\pi} \sum_{n=0}^{\infty} I_{n+2} C_{n} .
$$

The usual series expansion relative to $\beta$ is now expressed by the expansion relative to $C_{n}$ and $S_{n}$. The series expansion is now polynomial relative to $\rho$. At the central point of the line profile, i.e. at $\beta=0$, we get $\chi(0)=0, S_{n}=0$ and $C_{n}=L^{n} / n !$.

Summing up, we have obtained analytical series expansions which describe the spectral line profile centers of the hydrogenic species due to convolution of the Holtsmark, Lorentz and Doppler profiles at small argument values. The dependence on $\rho$ and $\varphi$ is incorporated in $C_{n}$ and $S_{n}$, and the polynomial dependence on $D^{2}$ - in the $I_{n+j}$ expressions. The series converges rapidly only at small values of $\rho$.

\section{SPECTRAL LINE PROFILES FOR THE INTERMEDIATE REGION}

For the intermediate region of the profiles of spectral lines we use a generalization of integral (19) with the integrand weighted by $x^{\mu-1}$ with positive $\mu$ values (Gradshtein \& Ryzhik 1962, p. 351, Eq. 3.462.1 and p. 510, Eqs. 3.953.1 and 2 ), obtained by the series expansion of the Holtsmark exponential factor. The analytical solution for these additives in this series expansion has the form

$$
I(\mu ; z)=\int_{0}^{\infty} x^{\mu-1} \exp \left(-D^{2} x^{2}-\gamma x\right) d x=2^{-\mu / 2} D^{-\mu} \Gamma(\mu) \Delta_{-\mu}(z),
$$

where, to simplify the formulae, instead of $\gamma$ we use the reduced complex argument

$$
z=\frac{\gamma}{\sqrt{2} D}
$$

and instead of the usual parabolic cylinder functions $D_{-\mu}(z)$ their modification by

$$
\Delta_{-\mu}(z)=\exp \left(\frac{z^{2}}{4}\right) D_{-\mu}(z)
$$

As it will be shown further, such modification enables to get rid of the exponential terms. 
Expressed via the confluent hypergeometric series $\Phi$, the functions $\Delta_{-\mu}(z)$ have the form (Gradshtein \& Ryzhik 1962, p. 1078, Eq. 9.240; Abramowitz \& Stegun 1972, p. 691, Eq. 19.12.3)

$$
\Delta_{-\mu}(z)=2^{-\mu / 2}\left\{\frac{\sqrt{\pi}}{\Gamma\left(\frac{\mu+1}{2}\right)} \Phi\left(\frac{\mu}{2}, \frac{1}{2} ; \frac{z^{2}}{2}\right)-\frac{\sqrt{2 \pi} z}{\Gamma\left(\frac{\mu}{2}\right)} \Phi\left(\frac{1+\mu}{2}, \frac{3}{2} ; \frac{z^{2}}{2}\right)\right\} .
$$

The complex confluent hypergeometric series are defined in the form (Gradshtein \& Ryzhik 1962, p. 1072, Eq. 9.210.1):

$$
\Phi(\alpha, \gamma, z)=\sum_{n=0}^{\infty} \frac{\alpha(\alpha+1) \ldots(\alpha+n)}{\gamma(\gamma+1) \ldots(\gamma+n)} \frac{z^{n}}{n !} .
$$

In the formulae of the present section, the parameters $\mu$ of the integrals $I(\mu ; z)$ incorporate the Holtsmark exponential factor via its series expansion.

Our next task is to find higher order parabolic cylinder functions using the recurrence formulae. The expressions $I(\mu ; z)$ are especially useful for the intermediate regions of line profiles, which are the most complicated in computational aspect and joins the spectral line central region with their wings. The parabolic cylinder functions $D_{-\mu}(z)$ are often expressed in somewhat modified form, namely

$$
U(\mu-1 / 2, z)=D_{-\mu}(z) .
$$

Similarly, we introduce the changed arguments in the modified hyperbolic cylinder functions

$$
\Upsilon(\mu-1 / 2, z)=\Delta_{-\mu}(z)
$$

Thus, semi-integer values of the parameter $\mu$ in $D_{-\mu}(z)$, which correspond also to semi-integer values of the parameter $\mu$ in the integral (52), acquire the opposite sign and the shifted value of the parameter, changing the semi-integer values of the parameter in the modified functions to the integer ones, and vice versa - the integer values of the parameter $\mu$ acquire the semi-integer values.

\section{RECURRENCE FORMULAE}

For the functions $U(\mu, z)$ the recurrence formulae are valid, which in the present study are expressed in the form

$$
U(\mu, z)=a_{\mu} U(\mu-1, z)+b_{\mu} U(\mu-2, z)
$$

where

$$
a_{\mu}=-\frac{z}{\mu-\frac{1}{2}}, \quad b_{\mu}=\frac{1}{\mu-\frac{1}{2}} .
$$

Thus

$$
\Upsilon(\mu, z)=a_{\mu} \Upsilon(\mu-1, z)+b_{\mu} \Upsilon(\mu-2, z) .
$$

From these equations for our problem we obtain the two chains of the recurrence formulae: for the half-integer values of $\mu$, reduced to the two lowest positive parameter as starting functions

$$
\Upsilon(\mu, z)=c_{\mu}^{\prime} \Upsilon\left(\frac{3}{2}, z\right)+d_{\mu}^{\prime} \Upsilon\left(\frac{1}{2}, z\right)
$$


and similarly for the integer values of $\mu$

$$
\Upsilon(\mu, z)=c_{\mu}^{*} \Upsilon(1, z)+d_{\mu}^{*} \Upsilon(0, z) .
$$

We find that, similarly to the recurrence formulae for $\Upsilon(\mu, z)$, the coefficients $c_{\mu}$ and $d_{\mu}$ also can be found by the recurrence formulae

$$
c_{\mu}=a_{\mu} c_{\mu-1}+b_{\mu} c_{\mu-2}
$$

and

$$
d_{\mu}=a_{\mu} d_{\mu-1}+b_{\mu} d_{\mu-2} .
$$

Now we have to specify the starting values of these coefficients for the given recurrence series. From the recurrence formula for $\Upsilon(5 / 2, z)$ we obtain

$$
c_{5 / 2}^{\prime}=a_{5 / 2}, \quad d_{5 / 2}^{\prime}=b_{5 / 2}
$$

and thereafter from the next recurrence formula for $\Upsilon(7 / 2, z)$ it follows that

$$
c_{7 / 2}^{\prime}=a_{5 / 2} a_{7 / 2}+b_{7 / 2}, \quad d_{7 / 2}^{\prime}=a_{7 / 2} b_{5 / 2} .
$$

Similarly, from the recurrence formula for $U(2, z)$ it follows that

$$
c_{2}^{*}=a_{2}, \quad d_{2}^{*}=b_{2}
$$

and from the next recurrence formula for $U(3, z)$

$$
c_{3}^{*}=a_{2} a_{3}+b_{3}, \quad d_{3}^{*}=a_{3} b_{2} .
$$

Thus, the chain of the recurrence formulae for needed integrals reduces a set of parabolic cylinder functions to their four lowest non-negative parameter functions, namely $\Upsilon(0, z), \Upsilon(1 / 2, z), \Upsilon(1, z)$ and $\Upsilon(3 / 2, z)$. It must be emphasized that these parabolic cylinder functions are expressed via two confluent hypergeometric functions, having low values of parameters and thus granting a rapid convergence rate of the expansion series.

The next task is to carry out summation over higher order parabolic cylinder functions, i.e., to derive the expression for the needed weighted sums of the integrals

$$
I(\mu ; z)=2^{-\mu / 2} D^{-\mu} \Gamma(\mu) \Upsilon(\mu-1 / 2, z) .
$$

Using the series expansion of the Holtsmark exponential factor $\exp \left(-x^{3 / 2}\right)$, we obtain for the parameter $\mu$ the values

$$
\mu=\mu_{k}=j+\frac{3 k}{2}
$$

where $j$ corresponds to the contribution from outside of the series expansion, and $3 k / 2$ corresponds to the series expansion of the Holtsmark exponential factor. The final expressions, we are seeking for as the sum of the terms of the integrated series expansion, can be expressed in the form

$$
Q_{j}(z)=\sum_{k=0}^{\infty} \frac{(-1)^{k}}{k !} I\left(\mu_{k} ; z\right) .
$$


Using the formula (67) the integrals can be written in the form

$$
Q_{j}(z)=\sum_{k=0}^{\infty} \frac{(-1)^{k} \Gamma\left(\mu_{k}\right) \Upsilon\left(\mu_{k}-1 / 2, z\right)}{k !\left(2 D^{2}\right)^{\mu_{k} / 2}} .
$$

These are the final expressions for integrals in the intermediate region of a spectral line.

The series expansion for $Q_{j}(z)$ rapidly converges if $D>1$. For stellar atmospheres this means that the convergence relative to $D$ is rapid for the rarefied higher atmospheric layers, where the Doppler width exceeds considerably the Holtsmark width.

The next task is to find suitable formulae for computation of the confluent hypergeometric functions. Generally speaking, this is a complicated problem, and for different values of these parameters and arguments different approximation procedures have been applied and different formulae have been derived.

The confluent hypergeometric series can be expressed in a very compact form:

$$
\Phi(\alpha, \gamma ; z)=\sum_{n=0}^{\infty} \frac{\alpha_{n}}{\gamma_{n}} \frac{z^{n}}{n !}
$$

where

$$
\alpha_{n}=\alpha(\alpha+1) \ldots(\alpha+n)=\prod_{i=0}^{n}(\alpha+i)
$$

and

$$
\gamma_{n}=\gamma(\gamma+1) \ldots(\gamma+n)=\prod_{i=0}^{n}(\gamma+i) .
$$

Consequently, we can write the recurrence formula as

$$
\frac{\alpha_{n}}{\gamma_{n}}=\frac{\alpha_{n-1}}{\gamma_{n-1}} \frac{(\alpha+n)}{(\gamma+n)} .
$$

For small values of the ratio $\alpha / \gamma$ the ratio $\alpha_{n} / \gamma_{n}$ at large values of $n$ tends to a finite value, $\alpha_{N} / \gamma_{N}$, and the following additive terms thereafter can be considered as belonging to corresponding exponential function. Thus, we can write

$$
\Phi(\alpha, \gamma ; z)=\frac{\alpha_{N}}{\gamma_{N}} \exp (z)+\sum_{n=0}^{N}\left(\frac{\alpha_{n}}{\gamma_{n}}-\frac{\alpha_{N}}{\gamma_{N}}\right) \frac{z^{n}}{n !}
$$

Numerical simulations have shown that a quite precise approximation can be obtained taking $N=150$. The relative error of the computed confluent hypergeometric function can be estimated as a ratio of the modules of the last term to the module of $\Phi$, i.e., as

$$
\varepsilon=\frac{\alpha_{N}}{\gamma_{N}} \frac{\left|z^{n}\right|}{n !} \frac{1}{|\Phi(\alpha, \gamma ; z)|} .
$$

The problem, how to fix the limits of acceptability of the derived formulae in the best way, deserves further study. 


\section{GENERAL DISCUSSION}

We have found analytical series expansions for the computation of spectral line profile functions for hydrogenic spectral line profiles, specified as a convolution of the Holtsmark, Lorentz and Doppler profile functions. The obtained formulae work well in the spectral line wings. In the central part of a line, to about the argument value $\Delta \nu_{S}$, the situation is much more complicated. Therefore, one of the most important circumstances is that each hydrogenic spectral line, due to the electrostatic field of plasma ions, splits into a large number of overlapping components extending to the line center. Such complicated spectral region probably exceeds the characteristic Stark line width $\Delta \nu_{S}$.

Similar result follows from the model microfield method computations, elaborated by Brissaud \& Frisch (1971), see also Brissaud et al. (1976), and applied for study of the hydrogenic spectral line profile functions by a number of investigators, especially for stellar spectra by Stehlé \& Hutcheon (1999). The model microfield method, based on the quantum electrodynamics, has been used by several authors, and it also takes into account that hydrogenic particles are affected by the temporally changing electric field strength of the adjacent ions. It has been assumed that the microfield is constant during a given time interval, and thereafter hydrogenic particles accommodate to some other constant value of the electric field strength. Such transitions are often called the 'kangaroo jumps'.

The process modifies central part of the line profile, and as follows from the model computations, it generates a central plateau, which extends to the maximum of the Holtsmark profile, but does not modify the line wings. An approximation, which satisfies these asymptotic features, can be obtained assuming that the microfield generates stochastic 'kangaroo jumps' in the electric field of the adjacent ions, generating transitions which give an additional Doppler distribution profile. For it we accepted the form $\exp \left(-\beta^{2} / 2\right)$.

Such distribution function is intrinsically bound with the Holtsmark distribution and modifies the convolved profile as the additional function, generating broadened Doppler profile, which removes the central dip in the line profile function. The factor $1 / 2$ in the exponent has been chosen from the elegance principle, because the Fourier transform of this function conserves the form. And last, but not least - such term increases the values of $D$ in the convolved profile, therewith facilitating the model computations.

As an example, in Figure 1 we demonstrate the model spectrum $\left(T_{\text {eff }}=16000\right.$ $\mathrm{K}, \log g=4)$ in the region of the hydrogen Balmer lines computed using the revised spectral line profile function. The $\mathrm{x}$-axis is given in the $\log \lambda[\mathrm{nm}]$ units, and the $\mathrm{y}$-axis is the reduced temperature $t_{\lambda}$ which has been computed adopting that the emergent Eddington flux (the flux per steradian) is equal to the Planckian black-body radiation intensity, $B_{\lambda}\left(t_{\lambda} T_{\text {eff }}\right)=H_{\lambda}$. The figure demonstrates also a smooth transition from the Balmer lines to the Balmer continuum, obtained by additional using of the dissolution fractions for the high hydrogen states.

In reality, physical processes acting on line profiles are very complicated, and their precise account hitherto is missing. Thus, a detailed analysis of total spectral line profile functions remains on agenda. Also the problem to determine reasonable limits for jumping from one formula to another remains to be solved. 


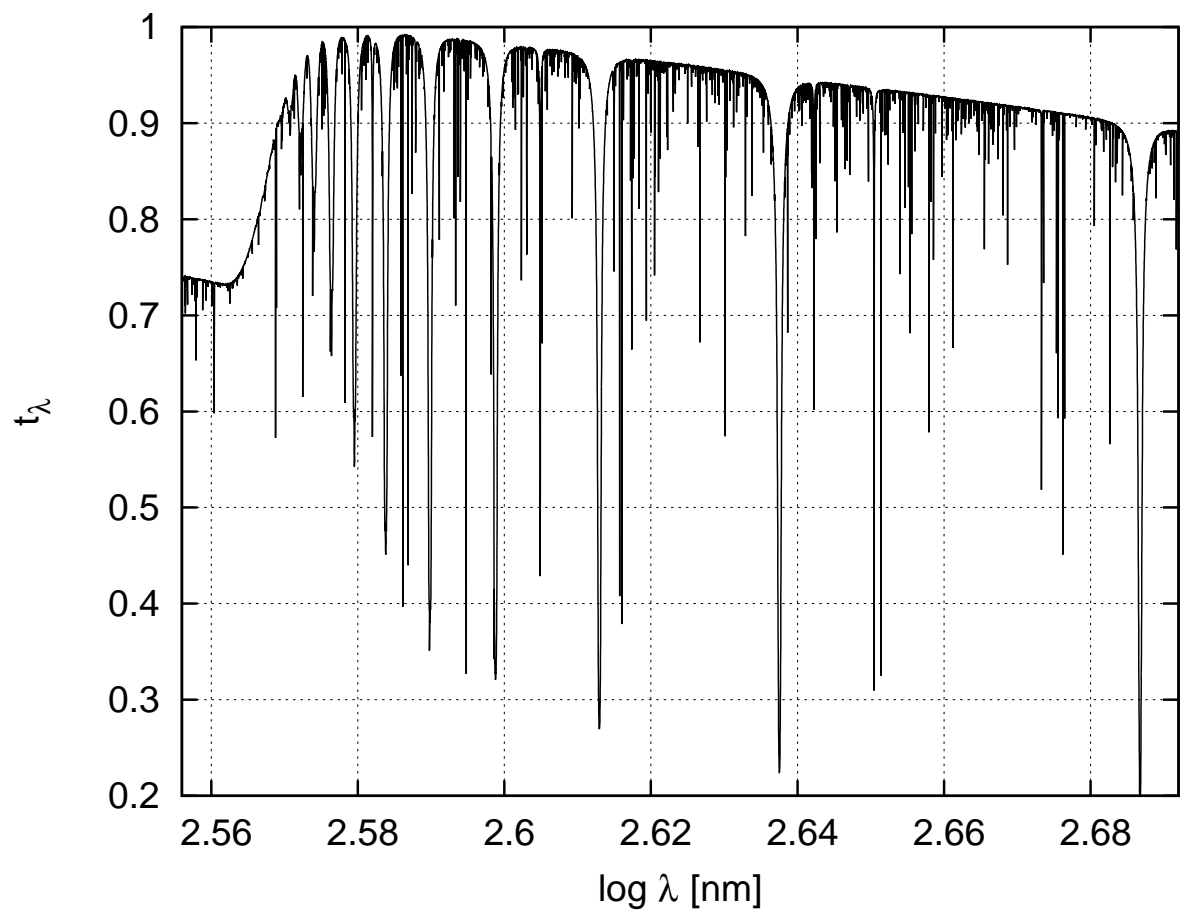

Fig. 1. The model spectrum $\left(T_{\text {eff }}=16000 \mathrm{~K}, \log g=4\right)$ in the region of the Balmer lines. The abscissa is $\log \lambda[\mathrm{nm}]$ and the ordinate is the reduced temperature $t_{\lambda}$, which corresponds to the black-body radiation.

ACKNOWLEDGMENTS. This work was supported by the research project SF0060030s08 of the Estonian Ministry of Education and Research.

\section{REFERENCES}

Abramowitz M., Stegun I. (eds), 1972, Handbook of Mathematical Functions

Brissaud A., Frisch U. 1971, JQSRT, 11, 1767

Brissaud A., Goldbach C., Léorat J., Mazure A., Nollez G. 1976, J. Phys. B: Atom. Molec. Phys., 9, 1129

Gradshtein I. S., Ryzhik I. M. 1962, Tables of Integrals, Series and Products, in Russian

Griem H. 1960, ApJ, 132, 883

Sapar A., Kuusik I. 1973, Tartu Astr. Obs. Publ., 40, 143

Sapar A., Poolamäe R., Sapar L. 2006, Baltic Astronomy, 15, 435

Sapar A., Aret A., Sapar L., Poolamäe R. 2009, New Astronomy Reviews, 53, 240

Sobelman I. I. 1963, Introduction to the Theory of Atomic Spectra, in Russian

Stehlé C., Hutcheon R. 1999, A\&AS, 140, 93 